\title{
The Neuropathic Pain Features in Psoriatic Arthritis: A Cross-sectional Evaluation of Prevalence and Associated Factors
}

\author{
Marco Di Carlo(i), Pietro Muto (D), Devis Benfaremo (i), Michele Maria Luchetti(i), \\ Fabiola Atzeni (iD, and Fausto Salaffi (iD)
}

\begin{abstract}
Objective. To evaluate the prevalence and factors associated with the neuropathic pain features in a cohort of patients with psoriatic arthritis (PsA).

Methods. A cross-sectional evaluation was conducted in consecutive patients having PsA with prevalent peripheral joint involvement, referred to 3 rheumatological centers. For each patient, a comprehensive assessment of disease activity, physical function, and disease effect was carried out. The presence of comorbid fibromyalgia syndrome (FMS) was evaluated. Acute-phase reactants were also recorded. The neuropathic pain features were investigated through the PainDETECT Questionnaire (PDQ). A logistic regression analysis was therefore conducted using the PDQ as the dependent variable.
\end{abstract}

Results. The final evaluation included 118 patients. A comorbid FMS was detectable in 30 of the 118 patients with PsA (25.4\%). Probable characteristics of neuropathic pain (PDQ $\geq 19$ ) were found in $30(25.4 \%)$ patients overall, ambiguous (PDQ > 12 and $<19)$ in $21(17.8 \%)$ patients, and unlikely $(\mathrm{PDQ} \leq 12)$ in $67(56.8 \%)$ patients. Using logistic regression analysis, the only independent variable among those investigated that could explain the neuropathic pain features was the presence of a comorbid FMS ( $p=0.0127)$. Excluding patients with comorbid FMS, an association with disability (measured by Health Assessment Questionnaire-Disability Index) emerges ( $\mathrm{p}=0.0489)$. In patients with PsA and comorbid FMS, PDQ scores were significantly higher than in patients without comorbid FMS.

Conclusion. Neuropathic pain features are common in patients with PsA, and the presence of pain sensitization (comorbid FMS) seems to be its main predictor. (First Release June 15 2020; J Rheumatol 2020;47:1198-1203; doi:10.3899/jrheum.190906)

Key Indexing Terms: PSORIATIC ARTHRITIS

\section{NEUROPATHIC PAIN FEATURES}

FIBROMYALGIA

The experience of pain is the characteristic that most unites chronic inflammatory joint diseases, the genesis of which is involved in multiple mechanisms ${ }^{1}$. The problem of chronic pain in inflammatory diseases is a much-debated area of research: while up to a few years ago it was considered only a symptom, at present there is increasing evidence that chronic pain is a disease in its own right ${ }^{2,3}$.

\footnotetext{
From the Rheumatological Clinic, Università Politecnica delle Marche, Ospedale "C. Urbani," Jesi (Ancona); Rheumatology Unit, Policlinico Universitario “G. Martino,” Università degli studi di Messina, Messina, Medical Clinic, Università Politecnica delle Marche, Ancona, Italy. M. Di Carlo, MD, Rheumatological Clinic, Università Politecnica delle Marche, Ospedale “C. Urbani”; P. Muto, MD, Rheumatology Unit, Policlinico Universitario "G. Martino," Università degli studi di Messina; D. Benfaremo, MD, Medical Clinic, Università Politecnica delle Marche; M.M. Luchetti, MD, Medical Clinic, Università Politecnica delle Marche; F. Atzeni, MD, Rheumatology Unit, Policlinico Universitario "G. Martino," Università degli studi di Messina; F. Salaffi, MD, PhD, Rheumatological Clinic, Università Politecnica delle Marche, Ospedale "C. Urbani."

Address correspondence to Dr. M. Di Carlo, Rheumatological Clinic, Università Politecnica delle Marche, Ospedale "C. Urbani,” Jesi (Ancona), Italy.E-mail: dica.marco@yahoo.it

Accepted for publication November 8, 2019.
}

In the field of inflammatory joint diseases, being able to correctly assess pain is essential because it is a variable that enters into the computation of the main disease activity indices. These include the 28-joint count Disease Activity Score and the Clinical Disease Activity Index for rheumatoid arthritis $(\mathrm{RA})^{4}$; the Ankylosing Spondylitis Disease Activity Score for ankylosing spondylitis (AS) ${ }^{5}$; or the Disease Activity index for PSoriatic Arthritis (DAPSA) for psoriatic arthritis (PsA) This is an important issue because disease activity indices represent a crucial variable both in daily clinical practice and in observational studies and clinical trials.

While the pain of inflammatory joint diseases could be the prototype of nociceptive pain, because it can fully meet the definition of the International Association for the Study of Pain (IASP; "pain that arises from actual or threatened damage to non-neural tissue and is due to activation of nociceptors" $)^{7}$, patients with chronic inflammatory joint diseases frequently complain of complex pain symptoms, with neuropathic characteristics (e.g., radiating pain, burning or tingling sensations) that can be regarded in the context of "mixed pain" states ${ }^{8}$.

Personal non-commercial use only. The Journal of Rheumatology Copyright ( 2020. All rights reserved. 
The presence of neuropathic pain features has already been studied in the field of RA and more widely in osteoarthritis $(\mathrm{OA})^{9,10,11,12,13}$. Regarding RA, it has been revealed that neuropathic pain features are present in at least $13 \%$ of patients, that these can be identified at an early stage of the disease, and that their presence impairs the achievement of remission at the 6-month followup period ${ }^{10}$. In OA, the prevalence of neuropathic pain is estimated to be around $23 \%{ }^{13}$, with significant persistence even when invasive treatment strategies such as total knee replacement are used ${ }^{12}$.

In axial spondyloarthritis (axSpA), both AS and nonradiographic axSpA, the presence of neuropathic pain features is just over $30 \%{ }^{14}$, and is associated with a reduced quality of life, poorer patient-reported outcome measures (PRO), and higher functional limitation ${ }^{15}$.

The only data related to the neuropathic pain features in PsA come from the DANBIO register ${ }^{16}$. In this Danish database, the presence of neuropathic pain was assessed through the PainDETECT questionnaire (PDQ). The researchers involved in that study investigated pain under multiple conditions of rheumatological interest. In the context of PsA, the presence of neuropathic pain features has been documented in $28 \%$ of patients, a higher percentage compared to both RA and axSpA.

The clinical variables associated with the presence of neuropathic pain features in PsA, to date, are not well known and have been poorly studied. On the basis of these assumptions, the objective of our study was to evaluate the prevalence and the clinical variables associated with the presence of neuropathic pain features in patients with PsA.

\section{MATERIALS AND METHODS}

Setting and patients. For the purpose of this study, consecutive patients with PsA from the outpatient clinics of 3 Italian rheumatological centers were included. Patients were enrolled from November 2018 to May 2019 and represented a sample of real-life clinical practice. The criteria for inclusion were adult patients with the presence of PsA with peripheral joint involvement (but patients with sacroiliitis or axial skeleton involvement were not excluded), diagnosed according to the ClASsification criteria for Psoriatic ARthritis (CASPAR) ${ }^{17}$. The exclusion criteria were the presence of active skin conditions other than psoriasis, the presence of inflammatory articular comorbidities (such as gout or calcium pyrophosphate crystal arthropathy), and the presence of coexisting neuropathic conditions demonstrated by instrumental examinations, such as entrapment neuropathies (e.g., carpal tunnel syndrome), cervical or lumbar radiculopathies, and polyneuropathies supported by any etiology.

Patients underwent a cross-sectional evaluation in which, in a single day, an objective musculoskeletal examination was performed in each center by an experienced rheumatologist (FS, FA, and MML) associated with the administration of PRO aimed at investigating the effect of PsA, functional status, and neuropathic features of pain. The questionnaires package was administered by a second rheumatologist (MDC, PM, and DB), blind to the results of the objective examination. For each patient, the demographic data, comorbidities, ongoing treatment, and acute-phase reactants were also recorded.

The study was conducted in accordance with the principles of the Helsinki Declaration and was approved by the local ethics committee (Comitato Etico Unico Regionale, number 0458 AS), and patients signed informed consent for anonymous data collection.
PsA measurements. The objective examination was focused on determining the tender joint count (0-68 joints), the swollen joint count (0-66 joints), and enthesitis assessed through the Leeds Enthesitis Index (LEI).

The first 2 variables, in addition to patient's global assessment of disease activity [0-10 numerical rating scale (NRS)], to NRS pain (0-10), and to C-reactive protein (CRP; in $\mathrm{mg} / \mathrm{dl}$ ), were used to calculate the DAPSA $^{6}$. DAPSA is a composite disease activity index that is PsA-specific. Currently the index is well recognized internationally. Through appropriate cutoffs, it allows establishment of the disease activity status: $\leq 4$ for remission (REM), $>4$ and $\leq 14$ for low disease activity (LDA), $>14$ and $\leq 28$ for moderate disease activity (MDA), and $>28$ for high disease activity $(\text { HDA })^{18}$

The LEI focuses on 6 entheseal sites (lateral epicondyles, medial femoral condyles, Achilles tendon insertions); it is easy to calculate because the final score is given by the sum of the enthesis involved, and it is considered an acceptable index because it is well correlated to the other disease activity measures ${ }^{19}$.

The 12-item Psoriatic Arthritis Impact of Disease (PsAID-12) has been used as a PRO indicative of the overall burden of a protean disease such as PsA in various health domains ${ }^{20}$. Composed of 12 NRS, it is easy to calculate, and the final value ranges from 0 to 10 , where higher scores indicate a more important disease burden for the patient. Some years ago our research group identified, through a multifactorial analysis, 2 main factors within the PsAID-12, which were called, respectively, PsAID Symptom Score, more involving joint symptoms, and PsAID Skin Score, more focused on the effect of psoriasis in health-related quality of life. For the purposes of our study we used these 2 subscales, calculated with the following formulas: PsAID Symptom Score $=($ NRS pain $\times 3+$ NRS fatigue $\times 2+$ NRS work and/or leisure activities $\times 2+$ NRS functional capacity $\times 2+$ NRS sleep disturbance $\times 2+$ NRS coping + NRS anxiety + NRS social participation + NRS depression $) / 15$; and PsAID Skin Score $=($ NRS skin problems $\times 2+$ NRS discomfort $\times 2+$ NRS embarrassment and/or shame) $/ 5^{21}$.

Functional ability was measured by the Health Assessment Questionnaire-Disability Index (HAQ-DI). The HAQ-DI assesses the degree of difficulty in performing common daily activities in 8 areas, with reference to the previous week. For each activity the patient is asked to respond on a 4-point scale (from $0=$ without difficulty, to $3=$ impossible), and for each functional area the highest value is considered. The final score is given by the average of the 8 values $^{22}$.

Neuropathic pain features assessment. PDQ was used in our study for the evaluation of neuropathic pain features. It was developed by the German Research Network on Neuropathic Pain over 10 years ago. The questionnaire has been validated in different clinical contexts such as postthoracotomy pain, neoplasms, low back pain, OA, fibromyalgia syndrome (FMS), but also in the field of inflammatory joint diseases. It is a completely self-administered questionnaire that can distinguish the nociceptive components from the neuropathic components of pain ${ }^{23}$. This symptom-based tool (no physical examination is required) investigates sensations related to the presence of neuropathic pain, such as allodynia, hyperalgesia, dysesthesia, and sudden pain. The PDQ is composed of seven 5 -point scales $(0=$ never, $5=$ very strongly) that investigate the qualitative characteristics of painful sensations (such as burning, tingling or prickling, pain to light touch, sudden pain attacks, cold or heat, numbness, slight pressure triggering pain) in body areas where the pain radiates (the presence of radiated pain has 2 points), indicated on a manikin. There is also a question that investigates the temporal trend of pain (score from -1 to 1 depending on the model selected). The final score can range from -1 to 38 ; for scores $\leq 12$ there is little chance $(<15 \%)$ that the pain has a neuropathic component. A score $\geq 19$ indicates that the pain has a strong probability of having a neuropathic component (>90\%), and finally a score between 13 and 18 indicates an ambiguous result ${ }^{24}$. Rifbjerg-Madsen and coworkers studied the PDQ psychometric properties (Rasch analysis and test-retest analysis) in a wide cohort of patients with inflammatory joint diseases (including PsA) and demonstrated acceptable properties in pain classification ${ }^{25}$.

Personal non-commercial use only. The Journal of Rheumatology Copyright () 2020. All rights reserved. 
Statistical analysis. Descriptive data are presented as mean and SD or as median and interquartile range. For the purpose of this study [i.e., to establish the influence of clinical and demographic variables (independent variables) on the presence of neuropathic pain features (dependent variable)], a logistic regression analysis was performed. Independent variables included age, body mass index (BMI), duration of articular disease, number of biologic agents (considering both the current and previous treatments), DAPSA, LEI, HAQ-DI, PsAID-12, PsAID Symptom Score, PsAID Skin Score, NRS pain, erythrocyte sedimentation rate, and CRP. In view of the important influence on painful symptoms, the presence of a comorbid FMS, diagnosed according to the criteria of the American College of Rheumatology (ACR; 2016) ${ }^{26}$, was also considered as an independent variable in the analysis. The dependent variable was represented by the PDQ. $P$ values were considered significant for values $<0.05$, and analyses were carried out with MedCalc 18.0.0 (MedCalc Software).

\section{RESULTS}

A total of 118 patients (42 men and 76 women, $35.6 \%$ and $64.4 \%$ of the population, respectively) with PsA were included, with a mean age of 57.67 (SD 12.54) years, and a mean BMI of 27.51 (SD 4.68) $\mathrm{kg} / \mathrm{m}^{2}$. The mean duration of articular disease was 7.97 (SD 7.02) years, and the mean duration of skin disease of 10.71 (SD 10.33) years. Descriptive statistics with reference to demographic and clinical variables are given in Table 1.

Sixty patients $(50.84 \%)$ were treated with at least 1 traditional disease-modifying antirheumatic drug (DMARD), of which $27(22.88 \%)$ were taking combination therapy. Specifically, 44 (37.28\%) took methotrexate, $17(14.40 \%)$ leflunomide, 15 (12.71\%) sulfasalazine, and 9 (7.62\%) cyclosporine.

In addition to traditional DMARD, 65 patients $(55.08 \%)$ were treated with a biological DMARD: 45 with an anti-tumor necrosis factor- $\alpha$ drug (21 etanercept, 13 adalimumab, 10 golimumab, and 1 certolizumab pegol), 15 $(12.71 \%)$ with secukinumab, and $5(4.23 \%)$ with ustekinumab. Thirteen $(11.01 \%)$ patients were taking apremilast.

Regarding antiinflammatory drugs, 14 patients (11.86\%) were taking low-dose corticosteroids $(<5 \mathrm{mg}$ /day prednisone), while 48 patients $(40.67 \%)$ were taking a nonsteroidal antiinflammatory drug (etoricoxib more frequently). Interestingly, $28(23.72 \%$ ) patients were taking drugs potentially used for neuropathic pain, including gabapentinoids (17 patients, $14.40 \%$ ), duloxetine (7 patients, $5.93 \%$ ), and tapentadol (4 patients, 3.39\%).

For disease activity, the mean DAPSA was 21.22 (SD 12.26), and 22 patients were in HDA, 68 in MDA, 25 in LDA, and 3 in REM.

Regarding the neuropathic pain features, the average PDQ was 13.15 (SD 8.07). As prevalence data, in the $25.4 \%$ of patients it was possible to identify likely neuropathic pain features (PDQ $\geq 19$ ), in the $17.8 \%$ ambiguous neuropathic pain features $(12<\mathrm{PDQ}<19)$, and in the $56.8 \%$ unlikely neuropathic pain features $(\mathrm{PDQ} \leq 12)$.

The logistic regression analysis showed that, among the variables under study, the only statistically significant correlation with the presence of neuropathic pain features was the presence of a comorbid FMS ( $p=0.0127$; Table 2), which was diagnosed in 30 patients $(25.42 \%)$. Most importantly, of those 30 patients with comorbid FMS, $28(93.3 \%)$ were women and only $2(6.7 \%)$ were men. Among the patients with comorbid FMS, there was also concentrated the use of drugs potentially for neuropathic pain (25 of the 30 patients mentioned above).

Table 1. Demographic and clinical variables of the whole cohort.

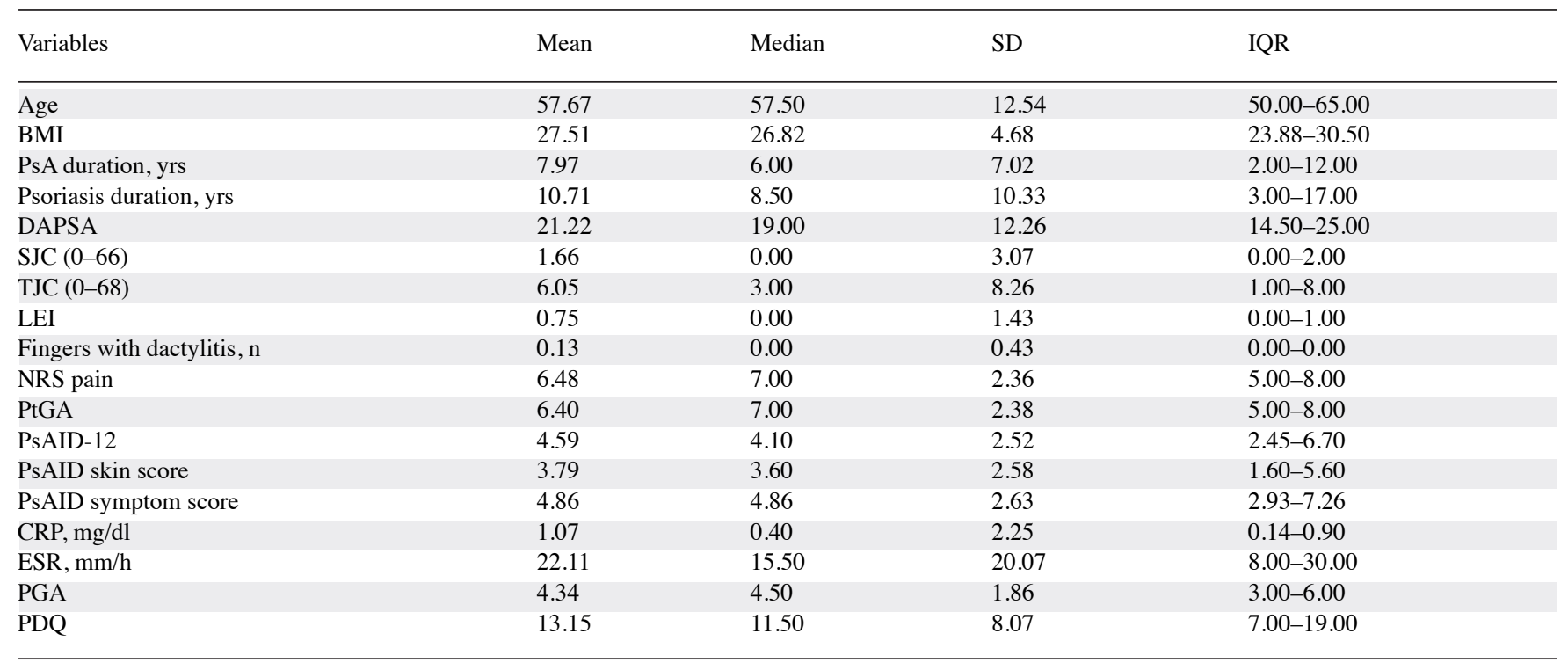

IQR: interquartile range; BMI: body mass index; PsA: psoriatic arthritis; DAPSA: Disease Activity Score for Psoriatic Arthritis; SJC: swollen joint count; TJC: tender joint count; LEI: Leeds Enthesitis Index; NRS: numerical rating scale; PtGA: patient's global assessment of disease activity; PsAID: Psoriatic Arthritis Impact of Disease; CRP: C-reactive protein; ESR: erythrocyte sedimentation rate; PGA: physician's global assessment of disease activity; PDQ: PainDETECT questionnaire.

Personal non-commercial use only. The Journal of Rheumatology Copyright $\subset$ 2020. All rights reserved. 
Table 2. Logistic regression analysis of the variables associated with neuropathic pain features (PDQ score $=$ dependent variable) in the whole cohort (118 patients).

\begin{tabular}{|c|c|c|c|c|}
\hline Variables & Coefficient & SE & Wald & $\mathrm{p}$ \\
\hline Age & 0.041895 & 0.040689 & 1.0601 & 0.3032 \\
\hline BMI & -0.10686 & 0.11523 & 0.8599 & 0.3538 \\
\hline PsA disease duration & -0.054908 & 0.065372 & 0.7055 & 0.4009 \\
\hline DAPSA & 0.060594 & 0.050190 & 1.4575 & 0.2273 \\
\hline HAQ-DI & 0.67158 & 0.79358 & 0.7162 & 0.3974 \\
\hline PsAID symptom score & 0.51869 & 0.37579 & 1.9052 & 0.1675 \\
\hline NRS pain & -0.55530 & 0.34046 & 2.6603 & 0.1029 \\
\hline ESR & -0.016822 & 0.026333 & 0.4081 & 0.5230 \\
\hline CRP & -0.23437 & 0.45772 & 0.2622 & 0.6086 \\
\hline FMS presence & 4.69187 & 1.96678 & 4.9153 & 0.0127 \\
\hline Constant & -5.03213 & 4.54624 & 1.2252 & 0.2683 \\
\hline
\end{tabular}

PDQ: PainDETECT questionnaire; SE: standard error; BMI: body mass index; bDMARD: biological disease-modifying antirheumatic drugs; PsA: psoriatic arthritis; DAPSA: Disease Activity Score for Psoriatic Arthritis; HAQ-DI: Health Assessment Questionnaire-Disability Index; LEI: Leeds Enthesitis Index; PsAID: Psoriatic Arthritis Impact of Disease; NRS: numerical rating scale; ESR: erythrocyte sedimentation rate; CRP: C-reactive protein; FMS: fibromyalgia syndrome.

It was decided to compare the categories of PDQ in patients without and with FMS. In this second set of patients, the scores of PDQ were statistically significantly higher (Table 3). A second logistic regression analysis was then performed, excluding patients with comorbid FMS. Analyzing the same variables in the remaining 88 patients, the only variable associated with the presence of neuropathic-like pain features (with some statistical significance) was HAQ-DI ( $\mathrm{p}=0.0489$; Table 4). In this group of 88 patients without comorbid FMS, it was possible to identify a prevalence of likely neuropathic pain features in 12 subjects $(13.63 \%)$, and of ambiguous neuropathic pain features in 17 subjects $(19.31 \%)$.

\section{DISCUSSION}

We have shown that the presence of neuropathic pain features concerns a substantial proportion of PsA patients, with a PDQ $\geq 19$ found in more than $25 \%$ of patients, and with a score between 13 and 18 in about 17\%. From a clinical point of view, this painful symptomatology with neuropathic features can be classified in the context of a comorbid FMS in most patients, largely attributable to the presence of pain sensitization. The results also show the clear prevalence of this kind of painful symptoms in the female population. Excluding comorbid FMS, the only variable associated with the presence of neuropathic-like pain features is disability, measured by HAQ-DI.

To the best of our knowledge, this is the first study that explored demographic and disease-specific clinical variables associated to the presence of neuropathic pain features in patients with PsA.

In the field of joint diseases, both inflammatory and degenerative, the mechanisms underlying the symptom pain have been the subject of intense research in recent years. In particular, there is a growing awareness that, alongside the nociceptive pathway, mechanisms involving a peripheral and central sensitization are involved ${ }^{27}$. Our results are in agreement with those collected by Rifbjerg-Madsen and colleagues who, from DANBIO register data, were the first to document the important prevalence of central pain

Table 3. PDQ categories in patients with PsA versus the presence or not of a comorbid FMS.

\begin{tabular}{|c|c|c|c|c|}
\hline No & 59 & 17 & 12 & $88(74.6 \%)$ \\
\hline Yes & $67(56.8 \%)$ & $21(17.8 \%)$ & $30(25.4 \%)$ & 118 \\
\hline Chi-square & 25.791 & & & \\
\hline DF & 2 & & & \\
\hline
\end{tabular}

PDQ: PainDETECT questionnaire; PsA: psoriatic arthritis; FMS: fibromyalgia syndrome; DF: degrees of freedom. 
Table 4. Logistic regression analysis of the variables associated with neuropathic pain features (PDQ score, dependent variable) excluding the patients with comorbid FMS (88 patients).

\begin{tabular}{lccc}
\hline Variables & Coefficient & SE & Wald \\
\hline BMI & & p & 0.04562 \\
Age & 0.043506 & 0.20368 & 1.8609 \\
No. bDMARD & 0.087252 & 0.063960 & 1.8790 \\
DAPSA & -1.01369 & 0.73950 & 0.7114 \\
HAQ-DI & -0.091035 & 0.10793 & 3.8793 \\
LEI & 3.74632 & 1.90208 & 0.1704 \\
PsAID skin score & -17.55864 & 6716.61311 & 0.3990 \\
PsAID symptom score & 0.72672 & 0.63157 & 0.0489 \\
NRS pain & -0.079835 & 0.60550 & 0.9979 \\
ESR & 0.65431 & 0.67047 & 0.2499 \\
CRP & -0.073561 & 0.049778 & 0.01738 \\
Constant & 0.061171 & 0.65208 & 0.9524 \\
\end{tabular}

PDQ: PainDETECT questionnaire; FMS: fibromyalgia syndrome; SE: standard error; BMI: body mass index; bDMARD: biological disease-modifying antirheumatic drugs; DAPSA: Disease Activity Score for Psoriatic Arthritis; HAQ: Health Assessment Questionnaire-Disability Index; LEI: Leeds Enthesitis Index; PsAID: Psoriatic Arthritis Impact of Disease; NRS: numerical rating scale; ESR: erythrocyte sedimentation rate; CRP: C-reactive protein.

mechanisms in patients with PsA, detecting a PDQ > 18 in $28 \%$ of subjects ${ }^{16}$.

It is interesting to note that among the other variables studied, neither the disease activity indices nor the effect of joint or skin disease is correlated to the presence of neuropathic pain features. Not including patients with comorbid FMS, only an association with disability emerges. The association between neuropathic pain and disability was already known, both in the field of OA and in the area of neurology 28,29 . In chronic inflammatory joint diseases, considering both PsA and RA, the correlation between HAQ-DI and PDQ has already been documented in the DANBIO register ${ }^{16}$. In our study we have made a step forward by evaluating the association in PsA patients without comorbid FMS.

It can be said that PDQ is a useful tool for recording the mechanisms of pain that go beyond synovial or entheseal inflammation. It is important to evaluate to make a proper diagnosis of pain and to avoid therapies that are often very expensive and potentially harmful. The debate remains open on whether the PDQ actually identifies the presence of peripheral neuropathic pain, or the central sensitization typical of FMS. On the other hand, the sensory profile of the 2 conditions is very similar ${ }^{30}$, and in addition, there is a considerable area of research that considers FMS a disease of the small fibers, because pathological alterations of the small fibers are present in about $49 \%$ of patients with FMS ${ }^{31}$.

Patients with neuropathic pain features meet the criteria for a comorbid FMS; this has already been documented for $\mathrm{RA}^{32}$. However, in patients with RA, there is a lower prevalence of neuropathic pain features than in $\mathrm{Ps}^{10,16,32}$. It can be speculated that the extent and severity of the psoriasis explain this difference, although there are no studies to date.

Also regarding knee OA, a community study with a large number of participants revealed that FMS is the main risk factor for the presence of neuropathic-like knee pain ${ }^{33}$. For this disease, the prognostic value of the presence of neuropathic pain features has been clearly demonstrated: higher scores of preoperative PDQ result in higher probability of persistence of chronic pain at 6 months from total knee replacement ${ }^{12}$.

A prognostic evaluation was also made in patients with early RA and it was documented that high scores of PDQ at baseline resulted in a very low probability of Boolean remission at 6 months $^{10}$.

The main limitations of our study are cross-sectional evaluation that did not allow prognostic evaluations, and the mild effect of psoriasis in our case study; skin disease may have an influence on certain pain descriptors of PDQ.

We documented the high prevalence of neuropathic pain features in patients with PsA (above 25\%), and it was demonstrated that this symptomatology is not attributable to disease activity, effect of disease, or demographic characteristics. Overall, the majority of patients with likely neuropathic pain features meet the 2016 ACR criteria for FMS Making a correct differential diagnosis of the pain symptom may be increasingly useful to avoid overtreatment with immunosuppressive drugs and using drug therapy appropriate to the pathophysiological mechanisms.

\section{REFERENCES}

1. Atzeni F, Masala IF, Salaffi F, Di Franco M, Casale R, Sarzi-Puttini P. Pain in systemic inflammatory rheumatic diseases. Best Pract Res Clin Rheumatol 2015;29:42-52.

2. Chiarotto A, Kaiser U, Choy E, Christensen R, Conaghan $\mathrm{PG}$, Cowern M, et al. Pain measurement in rheumatic and musculoskeletal diseases: where to go from here? Report from a Special Interest Group at OMERACT 2018. J Rheumatol 2019;46:1355-9.

3. Treede RD, Rief W, Barke A, Aziz Q, Bennett MI, Benoliel R, et al. A classification of chronic pain for ICD-11. Pain 2015;156:1003-7.

4. Dhaon P, Das SK, Srivastava R, Dhakad U. Performances of Clinical Disease Activity Index (CDAI) and Simplified Disease Activity Index (SDAI) appear to be better than the gold standard Disease Assessment Score (DAS-28-CRP) to assess rheumatoid arthritis patients. Int J Rheum Dis 2018;21:1933-9. 
5. Machado PM, Landewé RB, van der Heijde DM. Endorsement of definitions of disease activity states and improvement scores for the Ankylosing Spondylitis Disease Activity Score: results from OMERACT 10. J Rheumatol 2011;38:1502-6.

6. Smolen JS, Schoels M, Aletaha D. Disease activity and response assessment in psoriatic arthritis using the Disease Activity index for PSoriatic Arthritis (DAPSA). A brief review. Clin Exp Rheumatol 2015;5 Suppl 93:S48-50.

7. International Association for the Study of Pain. IASP terminology. [Internet. Accessed May 7, 2020.] Available from: www.iasp-pain. org/Education/Content.aspx?ItemNumber=1698\#Nociplasticpain

8. Freynhagen R, Parada HA, Calderon-Ospina CA, Chen J, Rakhmawati Emril D, Fernández-Villacorta FJ, et al. Current understanding of the mixed pain concept: a brief narrative review. Curr Med Res Opin 2019;35:1011-8.

9. Martins Rocha T, Pimenta S, Bernardo A, Bernardes M, Barbosa M, Lucas R, et al. Determinants of non-nociceptive pain in rheumatoid arthritis. Acta Reumatol Port 2018;43:291-303.

10. Salaffi F, Di Carlo M, Carotti M, Sarzi-Puttini P. The Effect of neuropathic pain symptoms on remission in patients with early rheumatoid arthritis. Curr Rheumatol Rev 2019;15:154-61.

11. Terry EL, Booker SQ, Cardoso JS, Sibille KT, Bartley EJ, Glover TL, et al. Neuropathic-like pain symptoms in a community-dwelling sample with or at risk for knee osteoarthritis. Pain Med 2020;21:125-37.

12. Kurien T, Arendt-Nielsen L, Petersen KK, Graven-Nielsen T, Scammell BE. Preoperative neuropathic pain-like symptoms and central pain mechanisms in knee osteoarthritis predicts poor outcome 6 months after total knee replacement surgery. J Pain 2018;19:1329-41.

13. French HP, Smart KM, Doyle F. Prevalence of neuropathic pain in knee or hip osteoarthritis: a systematic review and meta-analysis. Semin Arthritis Rheum 2017;47:1-8

14. Gok K, Cengiz G, Erol K, Ozgocmen S. Neuropathic pain component in axial spondyloarthritis and the influence on disease burden. J Clin Rheumatol 2018;24:324-7.

15. JiY Y, He Y, Nian X, Sun E, Li L. Inflammatory or neuropathic pain: characteristics and their relationship with disease activity and functional status in axial spondyloarthritis patients. Pain Med 2019;20:882-8.

16. Rifbjerg-Madsen S, Christensen AW, Christensen R, Hetland ML, Bliddal H, Kristensen LE, et al. Pain and pain mechanisms in patients with inflammatory arthritis: A Danish nationwide cross-sectional DANBIO registry survey. PLoS One 2017;12:e0180014.

17. Taylor W, Gladman D, Heliwell P, Marchesoni P, Mease P, Mielants H; CASPAR Study Group. Classification criteria for psoriatic arthritis: development of new criteria from a large international study. Arthritis Rheum 2006;54:2665-73.

18. Schoels MM, Aletaha D, Alasti F, Smolen JS. Disease activity in psoriatic arthritis (PsA): defining remission and treatment success using the DAPSA score. Ann Rheum Dis 2016;75:811-8.

19. Healy PJ, Helliwell PS. Measuring clinical enthesitis in psoriatic arthritis: assessment of existing measures and development of an instrument specific to psoriatic arthritis. Arthritis Rheum 2008;59:686-91.

20. Gossec L, de Wit M, Kiltz U, Braun J, Kalyoncu U, Scrivo R, et al; EULAR PsAID Taskforce. A patient-derived and patient-reported outcome measure for assessing psoriatic arthritis: elaboration and preliminary validation of the Psoriatic Arthritis Impact of Disease (PsAID) questionnaire, a 13-country EULAR initiative. Ann Rheum Dis 2014;73:1012-9.

21. Di Carlo M, Becciolini A, Lato V, Crotti C, Favalli EG, Salaffi F. The 12-item Psoriatic Arthritis Impact of Disease Questionnaire: construct validity, reliability, and interpretability in a clinical setting. J Rheumatol 2017;44:279-85.

22. Fries JF, Spitz P, Kraines RG, Holman HR. Measurement of patient outcome in arthritis. Arthritis Rheum 1980;23:137-45.

23. Freynhagen R, Tölle TR, Gockel U, Baron R. The painDETECT project - far more than a screening tool on neuropathic pain. Curr Med Res Opin 2016;32:1033-57.

24. Freynhagen R, Baron R, Gockel U, Tölle TR. painDETECT: a new screening questionnaire to detect neuropathic components in patients with back pain. Curr Med Res Opin 2006;22:1911-20.

25. Rifbjerg-Madsen S, Wæhrens EE, Danneskiold-Samsøe B, Amris K. Psychometric properties of the painDETECT questionnaire in rheumatoid arthritis, psoriatic arthritis and spondyloarthritis: Rasch analysis and test-retest reliability. Health Qual Life Outcomes 2017; $15: 110$

26. Wolfe F, Clauw DJ, Fitzcharles MA, Goldenberg DL, Häuser W, Katz RL, et al. 2016 revisions to the 2010/2011 fibromyalgia diagnostic criteria. Semin Arthritis Rheum 2016;46:319-29.

27. Salaffi F, Giacobazzi G, Di Carlo M. Chronic pain in inflammatory arthritis: mechanisms, metrology, and emerging targets-a focus on the JAK-STAT pathway. Pain Res Manag 2018;2018:8564215.

28. Polat CS, Doğan A, Sezgin Özcan D, Köseoğlu BF, Koçer Akselim S. Is there a possible neuropathic pain component in knee osteoarthritis? Arch Rheumatol 2017;32:333-8.

29. Poliakov I, Toth $\mathrm{C}$. The impact of pain in patients with polyneuropathy. Eur J Pain 2011;15:1015-22.

30. Koroschetz J, Rehm SE, Gockel U, Brosz M, Freynhagen R, Tölle TR, et al. Fibromyalgia and neuropathic pain--differences and similarities. A comparison of 3057 patients with diabetic painful neuropathy and fibromyalgia. BMC Neurol 2011;11:55.

31. Grayston R, Czanner G, Elhadd K, Goebel A, Frank B, Üçeyler $\mathrm{N}$, et al. A systematic review and meta-analysis of the prevalence of small fiber pathology in fibromyalgia: implications for a new paradigm in fibromyalgia etiopathogenesis. Semin Arthritis Rheum 2019;48:933-40.

32. Koop SM, ten Klooster PM, Vonkeman HE, Steunebrink LM, van de Laar MA. Neuropathic-like pain features and cross-sectional associations in rheumatoid arthritis. Arthritis Res Ther 2015;17:237.

33. Fernandes GS, Valdes AM, Walsh DA, Zhang W, Doherty M. Neuropathic-like knee pain and associated risk factors: a cross-sectional study in a UK community sample. Arthritis Res Ther $2018 ; 20: 215$ 